\title{
MAGNETIC TWEED CONTRAST IN FERROMAGNETIC SHAPE MEMORY ALLOYS
}

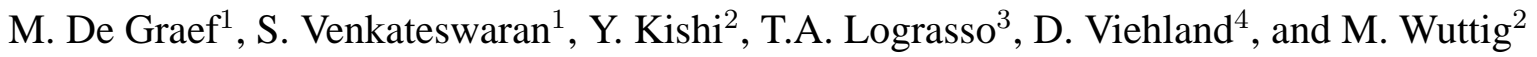 \\ ${ }^{1}$ Dept. of Materials Science and Engineering, Carnegie Mellon University, Pittsburgh, PA 15213 \\ 2 Dept. of Materials Science and Engineering, University of Maryland, College Park, MD 20742 \\ 3 Ames Laboratory, Iowa State University, Ames, IA 50011 \\ ${ }^{4}$ Dept. of Materials Science and Engineering, Virginia Tech, Blacksburg, VA 24060
}

Recently, ferromagnetic martensite, specifically ferromagnetic shape memory alloys (FMSMAs), have received renewed attention because of their large domain motion induced magnetostrictive strains. In the low temperature phase of these alloys, $90^{\circ}$ magnetic domain walls are simultaneously elastic twin boundaries. Above the temperature at which these alloys undergo a near second order martensitic transformation, their microstructure as observed by two-beam TEM methods, consists of the well known tweed contrast. This structure represents a random mixture of incipient twins of the martensitic phase. The transformation hysteresis of Co-Ni-Ga FMSMAs is quite small. In addition, the alloy is elastically soft similar to others that undergo a near second order martensitic transformation. Therefore, it is possible that magnetic tweed be observed in this alloy. This paper reports on its observation by conventional TEM, electron diffraction and Lorentz microscopy.

The TEM studies were carried out on a JEOL 4000EX microscope operating at $400 \mathrm{kV}$. This system is equipped with a Gatan imaging filter (GIF) which compensates for the magnification loss due to the increased focal length of the Lorentz imaging mode and also permits removal of inelastically scattered electrons to enhance the image contrast. Imaging of ferroelastic or structural domains was performed using the conventional bright field mode, whereas imaging of the ferromagnetic domain structure was performed using Lorentz microscopy (both Fresnel and Foucault imaging modes).

Fig. 1 shows a bright-field image of the (400) bend contour of $\mathrm{Co}_{0.50} \mathrm{Ni}_{0.205} \mathrm{Ga}_{0.295}$, taken at room temperature. The accompanying diffraction pattern was taken near the [110] zone axis orientation, and exhibits pairs of diffuse streaks in apparent [112] directions, typical for tweed modulated structures. The image reveals ferroelastic or structural domains. Tweed-like contrast can be seen, with a characteristic length scale of about $10 \mathrm{~nm}$. In this image, the cross-hatched tweed patterns are clearly evident on both sides of the bend contour. Zone axis patterns acquired for the [111] orientation reveal that the diffuse streaks lie along $\langle 110\rangle$-type directions. Fig. 2 shows again a bright field bend contour in the in-focus (b), under-focus (a), and over-focus (c) condition. While the bright band (arrowed) does not show any tweed contrast features in the in-focus image, the defocused images clearly reveal tweed-like contrast on a length scale of several tens of nanometers. Since the contrast only appears in the out-of-focus images, it must be caused by magnetic contributions to the electron phase shift. This has been confirmed using Foucault images, for which the displaced aperture again causes contrast to appear in the featureless region of Fig. 2b. Using the Transport-of-Intensity formalism [1] we have reconstructed the phase of the electron wave (shown in Fig. 2d). The components of the gradient of the phase are shown in (e) and (f), and reveal more clearly the modulated magnetic microstructure.

Tweed-like striations identical to those shown in Fig. 1 have previously been observed by TEM at temperatures above the transformation temperature, $\mathbf{M}_{S}$, which upon cooling below $\mathbf{M}_{S}$ condense into micron size twin structures, sometimes through an adaptive metastable phase. The known tweed structure in paramagnetic austensites is elastically driven. It can be anticipated that magneto-elastically driven magnetic tweed will equally condense into ferromagnetic martensite. The dual, magnetic and elastic, nature of the tweed demonstrated in Figs. 1 and 2 must be the result of magnetoelastic coupling. We will present theoretical considerations in support of the existence of magnetic tweed.

\section{References}

1. D. Paganin and K.A. Nugent, Phys. Rev. Lett. 80 (1998) 2586.

2. This work was supported by the U.S. National Science Foundation under grant numbers DMR- 
0095586 and DMR0095166, the Office of Naval Research, contracts No. MURI N000140110761, N000149910837 and N000140010849, and the Office of Basic Energy Sciences, Materials Sciences Division, of the U.S. Department of Energy under Contract Nos. W-7405-ENG-82 and DE-FG0201ER45893.

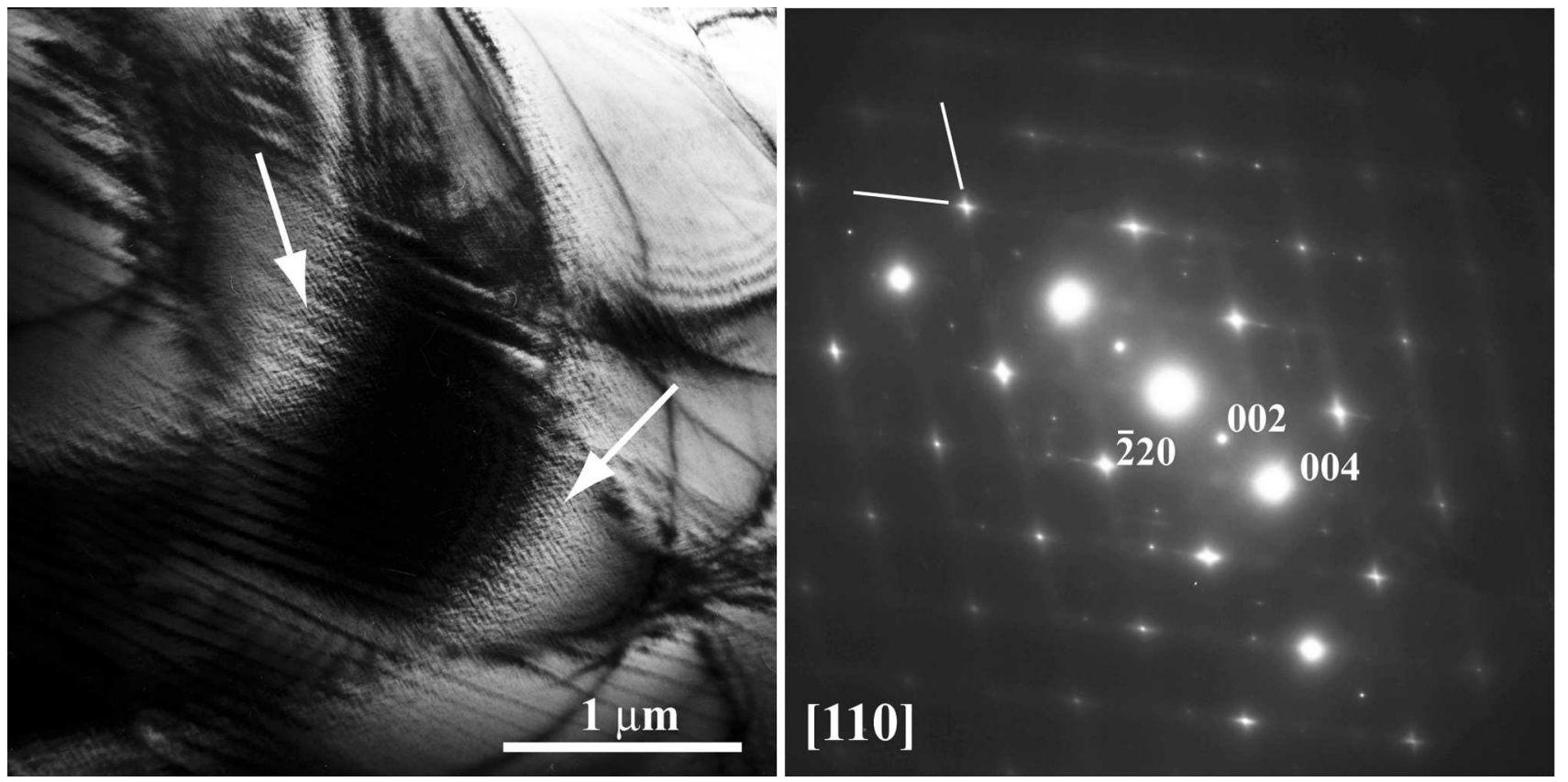

Fig. 1. conventional tweed contrast around the (004) bend contour of $\mathrm{Co}_{0.50} \mathrm{Ni}_{0.205} \mathrm{Ga}_{0.295}$. The near [110] zone axis diffraction pattern shows typical diffuse streaks in apparent [112] directions.

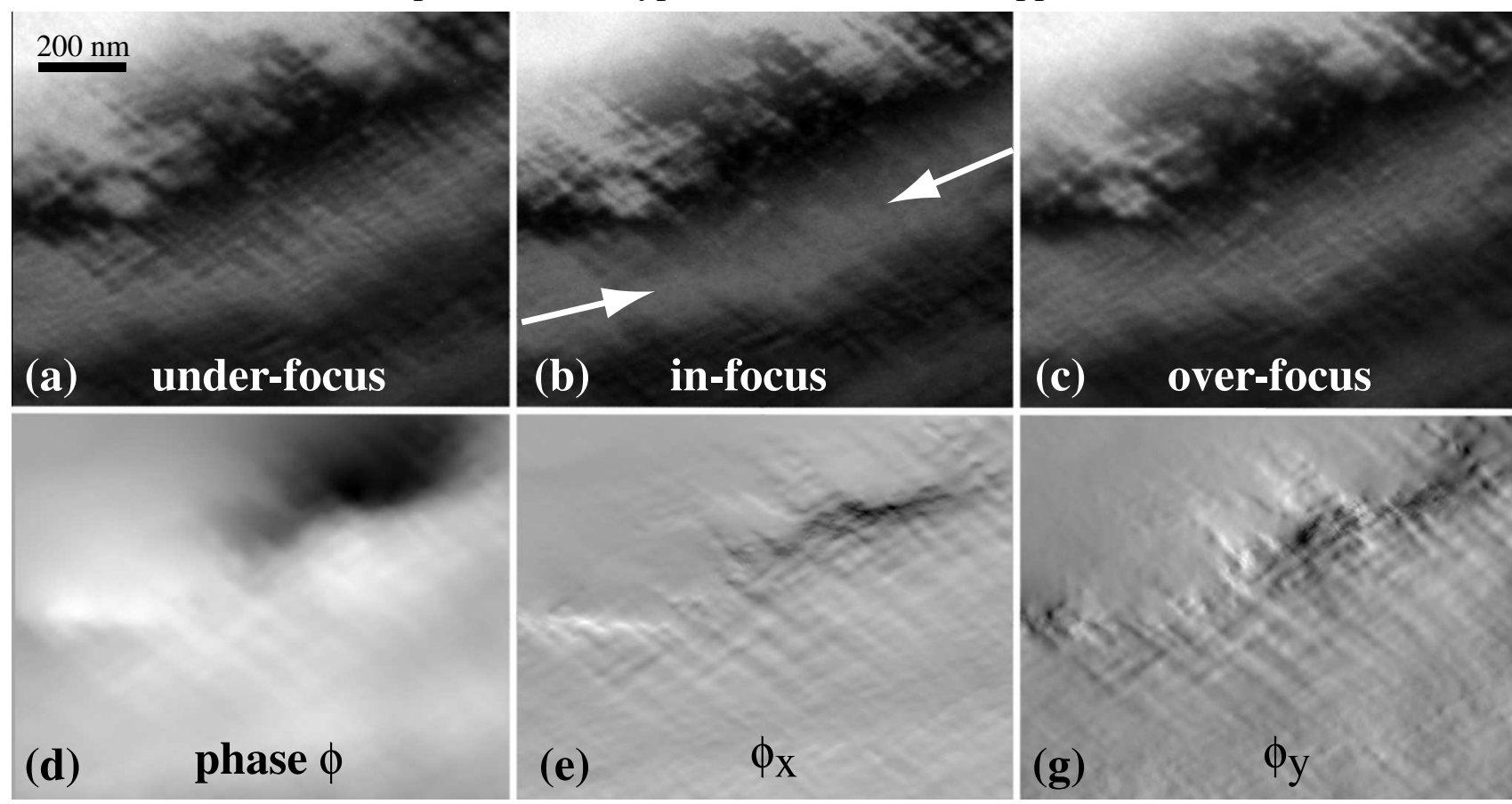

Fig. 2. Through-focus series (a-c) of a bend contour. Note the arrowed region in the in-focus image, which does not show any tweed contrast. The out-of-focus images do reveal tweed-like contrast. (d) represents the reconstructed phase, with the phase gradients $\phi_{X}$ and $\phi_{y}$ in (e) and $(g)$, resp. 